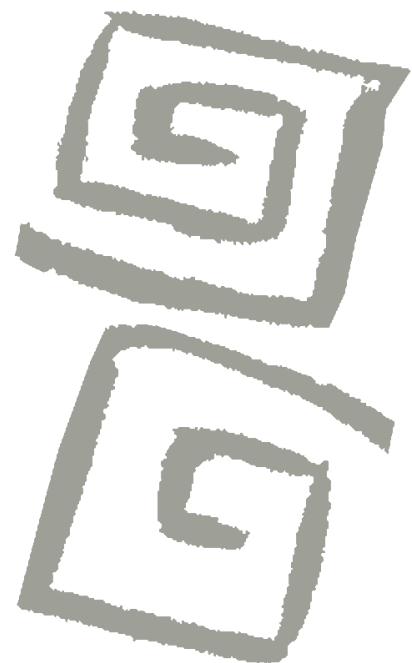

\title{
Asociaciones y disociaciones: agentes, discursos y controversias en torno a la hiperactividad infantil
}

\author{
Associations and dissociations: agents, discourses \\ and controversies surrounding child hyperactivity
}

Inmaculada Hurtado García ${ }^{1}$

'Doctora en Antropología Médica. Profesora, Departamento de Educación, Universidad CEU Cardenal Herrera. Elche, España. $\square$ (iD)
RESUMEN El trastorno por déficit de atención e hiperactividad (TDAH) genera debates que enfrentan a distintos agentes sociales con diferentes concepciones de la normalidad, la salud, el individuo y lo social. En este escenario de controversia, las madres y padres han tratado de mejorar las condiciones de vida de sus hijos desde diversos modelos de participación social en salud. Desde un abordaje cualitativo y etnográfico, se realizó un trabajo de campo entre 2013 y 2015 con el propósito de analizar la realidad asociativa española en torno al TDAH, así como otras iniciativas parentales individuales pero con ecos colectivos, para tratar de identificar las distintas relaciones con el conocimiento experto y los modelos de circulación de saberes que se dan en ellas, atendiendo a cómo configuran sus posicionamientos discursivos, establecen sus dinámicas colectivas y desarrollan sus acciones. La cualidad disputada del TDAH se manifiesta en modelos más complejos que el dual legos/expertos, así como en nuevas estrategias en la producción y colectivización del conocimiento facilitadas por Internet.

PALABRAS CLAVES Antropología Cultural; Trastorno por Déficit de Atención con Hiperactividad; Asociaciones de Salud Mental; Gestión del Conocimiento; España.

\footnotetext{
ABSTRACT Attention deficit hyperactivity disorder (ADHD) generates debates and confrontations among diverse social agents with different conceptions of normality, health, the individual and the social. In this scenario of controversy, parents have tried to improve the living conditions of their children through a number of models of social participation in health. Using a qualitative ethnographic approach, fieldwork was carried out from 2013 to 2015 with the purpose of analyzing the universe of organizations regarding ADHD in Spain as well as other individual parenting initiatives with collective repercussions. The work seeks to identify the different relationships with expert knowledge in existence and the models of knowledge circulation that take place within those relationships, focusing on the way they configure discursive stances, establish collective dynamics, and develop actions. The disputed character of ADHD is evidenced in models more complex than that of the expert/layperson duality, as well as in new strategies of production and collectivization of knowledge facilitated by the Internet.

KEY WORDS Cultural Anthropology; Attention Deficit Disorder with Hyperactivity, Mental Health Associations; Knowledge Management; Spain.
} 


\section{INTRODUCCIÓN}

El trastorno por déficit de atención e hiperactividad (TDAH) es uno de los diagnósticos psiquiátricos actuales más frecuentes en la infancia a nivel internacional. Se manifiesta como una combinación variable de dificultades para prestar atención y concentrarse, la presencia de un nivel alto de actividad inadecuado para la edad del niño y un deficiente control de impulsos ${ }^{(1)}$. Este exceso de inatención, hiperactividad e impulsividad ha sido descrito en la literatura desde el siglo XIX, recibiendo distintos nombres según qué manifestación de conducta destacara cada autor. En 1902, Still(2) introduce la etiqueta diagnóstica más similar a la descripción actual del síndrome. Aunque también se contempla en adultos por su carácter de condición crónica, ha generado más controversia en su aplicación a menores.

La hiperactividad se ha convertido en un marco comprensivo habitual del comportamiento de la infancia contemporánea occidental, así como un lugar común en el lenguaje de la cotidianeidad. De hecho, esta etiqueta se ha popularizado y se aplica de manera informal y anticipatoria en niños, cuya actividad es entendida por los adultos como excesiva, independientemente de que exista o no un diagnóstico. Al igual que sucede con la depresión o la ansiedad, la terminología diagnóstica ha pasado a ser usada por la población en general -más allá de pacientes y profesionales-, para interpretar, regular y mediar las experiencias y la comprensión del $\mathrm{yo}^{(3)}$. En este caso, la hiperactividad y su comprensión como trastorno han permitido resignificar y dar sentido, especialmente a las familias, al desajuste del comportamiento infantil con las normas sociales.

A pesar de la amplificación de la etiqueta de "hiperactividad", o precisamente por ello, hay un debate abierto sobre su consistencia como categoría diagnóstica ${ }^{(4,5)}$, que ha desembocado en la falta de consenso social con el relato biomédico. Resulta un caso paradigmático en el que podemos observar dos modelos polarizados en la interpretación de los malestares: el de la condición, sustentado por el enfoque biomédico, que describe realidades individualizadas y somáticas; y el de la mirada biopolítica, que destaca la naturaleza sociocultural de algunos de estos malestares, especialmente, de los psiquiátricos. En el primero, el nudo del desajuste se sitúa en el individuo, en sus conductas inapropiadas y las consecuencias que ello acarrea para él y su entorno; mientras que, en el segundo, se visibiliza la estrategia biomédica por la que los síntomas se reconvierten en signos y permiten, de este modo, desocializar, universalizar y neutralizar los malestares ${ }^{(6)}$.

El primer modelo vendría representado, en este caso, por el referente más utilizado a nivel internacional en salud mental: el Manual diagnóstico y estadístico de los trastornos mentales (DSM, por su sigla abreviada en inglés) que publica la Asociación Americana de Psiquiatría (APA). En su quinta y última edición, se afirma que el TDAH se caracteriza por un patrón persistente de comportamiento que se da en múltiples entornos, ejemplificados en el hogar y la escuela. Se considera que el trastorno está presente cuando las conductas de inatención, hiperactividad e impulsividad tienen mayor frecuencia e intensidad de lo habitual, según edad y desarrollo de la persona, y estas interfieren de forma significativa en el rendimiento escolar o laboral, y en sus actividades cotidianas ${ }^{(7)}$. Actualmente, el TDAH suele tratarse con psicofármacos, así como con terapias cognitivo-conductuales, en las que es crucial la colaboración de familias y de los docentes.

Desde el enfoque biopolítico se advierte, principalmente, la galopante patologización de la infancia y de la medicamentación que implica la creación de este trastorno ${ }^{(8)}$. Estas voces contemplan el TDAH como uno de los ejemplos de transferencia al ámbito médico del desajuste a la vida social, en concreto a la vida familiar y escolar, y una de las expresiones de la medicalización de la educación que más ha calado en el imaginario de los diferentes agentes educativos, sean padres, docentes o instituciones. Una de sus manifestaciones sería la oscilación de las tasas de prevalencia, según estudios 
y áreas geográficas, que vendría a demostrar la relevancia de los factores socioculturales y las tradiciones médicas en la interpretación y tratamiento de las conductas relacionadas con el TDAH ${ }^{(9,10)}$. Así, hay estudios que señalan que la prevalencia global del TDAH es de $5,29 \%$ en niños en edad escolar ${ }^{(11)}$. En la Unión Europea sería de un 5\% (3,3 millones) entre niños y adolescentes ${ }^{(12)}$, mientras que un estudio del año 2012 revela que la prevalencia en España sería de un 6,8\% ${ }^{(13)}$.

En este escenario de disenso, las personas afectadas y los familiares no solo se agrupan para elevar sus demandas de salud, sino que juegan un papel fundamental como agentes sociales activos que se posicionan y actúan según sus necesidades definidas en un contexto concreto. Las asociaciones de salud y los grupos de ayuda mutua son entidades formadas por personas afectadas por problemas de salud y/o por familiares de estas. Su formación viene motivada por múltiples causas pero, común a todas, es la de buscar soluciones a problemáticas mal atendidas $^{(14)}$ y de una creciente reivindicación de una participación más activa en los diseños de políticas y prestaciones de salud. En consecuencia, tanto las asociaciones de salud como los grupos de ayuda mutua tienen, al mismo tiempo, funciones asistenciales y reivindicativas. En muchos casos, conseguir la visibilidad social de una problemática marginada es la principal razón de ser de tales organizaciones y la que más determina su trayectoria y participación a partir de la producción de conocimientos ${ }^{(14,15)}$.

El interés en los grupos de pacientes, el activismo sanitario y los movimientos sociales en torno a la investigación médica es un área de estudio en la que científicos sociales han tratado de establecer clasificaciones y tipologías según las relaciones de estos con el sistema de salud y sus profesionales. Una de estas tipologías es la trazada por Rabeharisoa y Callon ${ }^{(15)}$, que permite clasificar las asociaciones de pacientes en auxiliares, cooperantes y opositoras, según sus diferentes maneras de implicarse y aliarse con el conocimiento experto y sus profesionales. Estas clasificaciones permiten hacer una primera aproximación desde la relación con el discurso experto, pero no agotan la enorme variedad de grupos, movimientos y dinámicas de participación que incluyen, entre otros, aquellos "virtuales" que surgieron en Internet o aquellos que actúan en nombre de otros grupos de pacientes ${ }^{(16)}$.

\section{ASPECTOS METODOLÓGICOS}

Este trabajo se enmarca en una investigación colectiva más amplia que aborda diferentes modelos de articulación entre organizaciones de pacientes, profesionales médicos y otros actores implicados, y el análisis de casos de saberes legos que permean la práctica científica ${ }^{(17,18,19,20)}$, y centra su interés en la experiencia colectiva de madres y padres de hijos con TDAH en el contexto español, tanto desde el movimiento asociativo como desde otras estrategias más singulares, pero que también consiguen tener una resonancia social destacable y permiten tejer redes de conocimiento. Las asociaciones de salud y los grupos de ayuda mutua se articulan desde la interacción social según entornos socioculturales, políticos y sanitarios específicos para hacer frente a sus necesidades y elevar sus demandas de manera situada. Por ello, para entender la configuración, causas y acciones de los grupos en torno al TDAH, estos necesitan ser situados en los contextos nacionales en los que se desarrollan y conforman, tal y como ya se ha hecho en anteriores estudios sobre grupos de padres en distintos países europeos como Francia, Irlanda, Italia y Reino Unido $^{(4,21,22,23)}$. A partir de las diferentes iniciativas colectivas de madres y padres de niños diagnosticados con TDAH, trataremos de mostrar sus modelos y estrategias de participación en salud, sus procesos de (des)identificación con el conocimiento experto y las actividades de producción de conocimiento desarrolladas en un escenario de controversia entre modelos explicativos.

Se ha seguido una metodología etnográfica consistente en la realización de trabajo de campo a lo largo de dos años (2013-2015) 
en los cuales se han identificado los agentes sociales implicados y se han seguido los discursos y debates en los medios de comunicación y redes sociales. En cuanto a las estrategias de movilización de madres y padres, se ha seguido de cerca la actividad de la Asociación de Alicante para el Déficit de Atención con o sin Hiperactividad (AADAH) y de la Federación Española de Asociaciones de Ayuda al Déficit de Atención e Hiperactividad (FEAADAH), organismo al que pertenece y que aglutina a gran parte de las asociaciones de TDAH en el Estado español. Se ha participado en charlas y cursos de formación, organizados para miembros y gente externa, se ha recopilado información de sitios web y publicaciones, y se ha entrevistado en profundidad a la presidenta del grupo local.

Como contrapunto de las adherencias más biomédicas y disociado del movimiento asociativo, también se entrevistó en profundidad al padre de un niño diagnosticado con TDAH y autor del blog "Yo también amo a alguien con... ¿TDAH?", a partir del cual se articulan otras redes de saberes en las que participan legos y expertos.

Las personas entrevistadas firmaron un consentimiento informado en el que aceptaban participar de la investigación, luego de haber recibido información sobre sus objetivos y el destino de la información recabada. Prescindiremos de los nombres propios de las personas entrevistadas, a pesar de que manifestaron no tener inconveniente en que se hicieran públicos, dada la visibilidad de la posición que ocupan.

\section{EL DISCURSO EXPERTO: PROTOCOLOS Y MANIFIESTOS}

EI TDAH es una categoría médica reconocida en los principales sistemas clasificatorios de enfermedades y por parte de organismos internacionales como la Organización Mundial de la Salud (OMS) y la Unesco, que genera debates a nivel internacional, tanto dentro como fuera del ámbito biomédico ${ }^{(24)}$. Las principales críticas vertidas a la expansión del diagnóstico de TDAH señalan su falta de rigor ante la ausencia de biomarcadores fiables que evidencien la causalidad; su ambigüedad, dado que la detección está basada mayormente en la observación de docentes y padres; su aplicación predictiva y no preventiva; y la utilización de un tratamiento, que incluye la administración de psicoestimulantes a menores, y que hace sospechar de los intereses farmacéuticos a los que sirve ${ }^{(25)}$. El debate sobre el diagnóstico es a menudo establecido de modo categórico respecto de su existencia y cualidad de real. El discurso legitimado biomédicamente afirma que existe una entidad clínica diferenciada y con una etiología orgánica determinada de carácter neurobiológico, mientras que otros no reconocen la categoría específica y consideran que se trata de una agrupación sintomática sin una clara etiología, que se manifiesta en diferentes estructuras de la personalidad y que responde a múltiples factores (biológicos, psicológicos, familiares y socioeducativos) ${ }^{(26)}$. Distintos profesionales de la educación y del sector "psi" también se oponen a las explicaciones médicas/biológicas de las dificultades derivadas del comportamiento infantil ${ }^{(27)}$ y cuestionan la complicidad de la escuela como lugar de producción de trastorno ${ }^{(28)}$.

No es objeto de este artículo problematizar cómo se establece el conocimiento científico del TDAH, su estructura nosológica, los criterios diagnósticos utilizados o dónde se inserta su práctica clínica. Sin embargo, estos debates son el escenario que moldea las funciones, objetivos y demandas del movimiento asociativo y de sus exilios. De estas críticas principalmente se han hecho eco en España profesionales de la salud ${ }^{(29)}$, la educación ${ }^{(30)}$ y la comunicación ${ }^{(31)}$. Por otro lado, quienes refrendan y atienden los casos de TDAH desde el modelo propuesto por el DSM y su concepción como trastorno de origen neurobiológico de inicio en la edad infantil proceden, fundamentalmente, de determinadas especialidades médicas (psiquiatría, neurología y pediatría), del ámbito psicológico clínico, a través de gabinetes psicopedagógicos y 
servicios específicos, de colectivos de afectados y familiares $y$, en menor medida, del campo educativo. Es decir, profesionales de las mismas disciplinas pero desde distintos posicionamientos. Otros de los agentes sociales implicados que juegan un papel fundamental, aunque aquí no podremos desarrollarlo, son los laboratorios farmacéuticos y las fundaciones, a menudo vinculados entre sí, que colaboran en la difusión del conocimiento sobre el TDAH en el dominio público, y participan activamente en las actividades de las asociaciones desde el patrocinio y la dotación de recursos de todo tipo a las personas afectadas y familiares.

El TDAH en España es refrendado por instituciones médicas, educativas y políticas a través de la publicación de guías, protocolos, normativas de aplicación y reconocimientos legislativos a nivel estatal, así como en las distintas comunidades autónomas, dadas sus competencias en materia de sanidad y educación. Estos se consideran logros del trabajo desempeñado por las asociaciones en la orientación de las necesidades de los niños con TDAH y en su demanda por obtener respuestas eficaces que garanticen los derechos de sus hijos a ser atendidos como precisan. La colaboración de las instituciones con la FEAADAH y las distintas asociaciones se manifiesta en su inclusión como consultores, revisores y destinatarios de los distintos documentos publicados como respuesta a sus demandas. La inclusión expresa del TDAH en la Ley Orgánica de Mejora de la Calidad Educativa en 2013, conjuntamente con las medidas de atención y escolarización del alumnado con dificultades específicas de aprendizaje, viene a ser un buen ejemplo. El reconocimiento de las necesidades específicas de apoyo educativo del alumnado con TDAH fue aprobado por una mayoría en el Congreso de los Diputados y, por otro lado, seis comunidades autónomas han establecido protocolos de actuación coordinados a nivel autonómico entre Sanidad, Educación y, en su caso, Servicios Sociales, para el diagnóstico y tratamiento del TDAH en España.

Estas guías y protocolos tienen la voluntad de establecer consensos y unificar prácticas clínicas en un escenario de controversia, en el que las asociaciones reclaman activamente reducir la incertidumbre de profesionales y de familias para protocolizar itinerarios terapéuticos. Sin embargo, otro de los efectos ha sido el de aglutinar y visibilizar a las distintas asociaciones médicas y entidades sociales más críticas que, a propósito de estos documentos, han demandado su modificación o su retirada. Es precisamente en el ámbito sanitario, a diferencia del educativo, donde más voces se han alzado para rechazar la tendencia implícita hacia un modelo único de comprensión y tratamiento del TDAH frente a otros marcos explicativos y opciones terapéuticas. Así, los debates y los posicionamientos de los distintos profesionales acreditados han alcanzado la esfera pública a partir de las publicaciones institucionales, de los manifiestos que las contestan y de los contramanifiestos profesionales y manifestaciones institucionales que las refrendan.

En 2010, se publicó la primera Guía de práctica clínica sobre el trastorno por déficit de atención con hiperactividad (TDAH) en niños y adolescentes por parte del entonces Ministerio de Sanidad, Política Social e Igualdad ${ }^{(32)}$. El objetivo era unificar criterios diagnósticos y servir de referente en la toma de decisiones sanitarias en el ámbito del Sistema Nacional de Salud español ante el problema de salud pública que supone el TDAH, dada su alta prevalencia y sus enormes consecuencias en la vida del paciente. Este documento contó con la revisión externa, entre otros, del presidente de la FEAADAH y, a pesar de ser una guía clínica, explicitaba entre sus pretensiones ser un instrumento útil para la población, los profesionales sanitarios y de educación.

Esta guía, junto con el Protocolo para el manejo del TDAH infanto-juvenil en el sistema sanitario catalán, elaborado por parte del Departamento de Salud de Cataluña, han sido algunos de los documentos más cuestionados. Este último, publicado el 6 de mayo de 2015, se implementó con el objetivo de favorecer la equidad en la atención a la población con trastorno por déficit de atención con o sin hiperactividad (TDAH) y dar respuesta a 
la petición de las asociaciones de familiares. En él se ofrecen diez recomendaciones para reducir la variabilidad en el diagnóstico y el tratamiento del TDAH, así como recomendaciones para la coordinación con el sistema educativo y el trabajo con familias.

Las críticas vertidas hacia este protocolo desembocaron en la publicación el 29 de junio de ese mismo año del manifiesto Para un consenso clínico del TDAH frente al actual diagnóstico estadístico o juicio evaluativo de control administrativo, que ha sido suscrito por 2.358 firmas y ha recibido 122 adhesiones institucionales. En él, asociaciones de profesionales, docentes, trabajadores sociales y, únicamente, una de familiares han pedido la retirada del protocolo. Algunas de estas entidades son la Asociación Española de Neuropsiquiatría, la Asociación Nacional de Enfermería de Salud Mental, la Federación de Asociaciones para la Defensa de la Sanidad Pública o la Sociedad Española de Psicología Analítica. Tras la firma de este documento, el 22 de julio de 2015 se publicó un contramanifiesto de apoyo al protocolo, considerando que recoge la evidencia científica sobre este trastorno. Este fue firmado, entre otras, por la Sociedad Catalana de Psiquiatría y Salud Mental, la Sociedad Catalana de Psiquiatría Infantojuvenil, la Sociedad Catalana de Pediatría de la Academia de Ciencias Médicas de Cataluña y Baleares y la Sociedad Catalana de Especialistas en Psicología Clínica. En estas facciones advertimos especialidades y enfoques distintos de la salud. Por un lado, los especialidades de corte más social y enfoques psicodinámi$\cos \mathrm{y}$, por otro lado, las especialidades "psi" con enfoques más cognitivo-conductuales y del neurodesarrollo, que privilegian las intervenciones sobre el comportamiento infantil junto con la medicación ${ }^{(33)}$.

Algunas de las críticas citadas más compartidas son la falta de rigor y la excesiva frecuencia de diagnóstico, así como la minimización de los riesgos del tratamiento farmacológico. Pero, de manera más concreta y situada, se defienden los sistemas públicos y los marcos culturales propios en un momento de fuertes recortes sanitarios y sociales, denunciando la tendenciosidad y los conflictos de interés implícitos de los profesionales de la sanidad privada que las han elaborado; reivindicando la utilización del referente diagnóstico europeo (CIE), publicado por la OMS, en lugar de los del DSM, menos restrictivos y gestados en EE.UU. La disputa de modelos de comprensión del TDAH que se despliegan en estas pugnas influirá enormemente en la función de las asociaciones, en las adhesiones a unas $u$ otras redes de expertos, y en la circulación del conocimiento entre los distintos grupos.

\section{EL MOVIMIENTO ASOCIATIVO EN TORNO AL TDAH}

La movilización social en torno al TDAH está formada mayormente por asociaciones diseminadas en todo el territorio español. La mayoría de ellas fueron fundadas por madres y padres que, en calidad de coafectados, se agrupan no solo para reclamar derechos y recursos para sus hijos sino para legitimar una experiencia de malestar que consideran que no es tratada adecuadamente y que además es cuestionada por las voces críticas, por algunos docentes en la praxis escolar, y por aquellos que los responsabilizan de un ejercicio de crianza falto de dedicación a los hijos, excesivo en sus expectativas y delegado a la voz experta ${ }^{(34)}$. En este sentido, el ámbito asociativo ha permitido organizar a madres y padres de niños con TDAH para hacer colectivas sus demandas, para negociar sentidos que exorcicen la culpa otorgada e incardinarse en redes de expertos con quienes articular su comunidad epistémica(35).

Gran parte de estas asociaciones están agrupadas en la FEAADAH, una asociación sin ánimo de lucro de carácter nacional que, desde el año 2002, se dedica a promover la investigación del TDAH y colaborar en áreas educativas, de atención social y científicas, con entidades consagradas al estudio y atención de este trastorno. Es, además, socia fundadora y miembro activo de la red europea de asociaciones de atención al TDAH, ADHD-Europe. Actualmente hay 84 
asociaciones federadas en el territorio español, aunque su concentración es más alta en áreas urbanas. Es un número considerable si lo comparamos, salvando las diferencias demográficas, con las existentes en otros países, como los 20 grupos locales en Italia ${ }^{(21)}$, las 25 asociaciones federadas en el INCADDS en Irlanda o los 22 grupos de HyperSupers en Francia ${ }^{(36)}$. El número de asociaciones en torno al TDAH podría explicarse a partir de las diferencias autonómicas, como lugares situados de vindicación, pero también por la dimensión de proximidad, cuidado emocional, intercambio de experiencias y legitimación social que supone el grupo de apoyo en un contexto de cuestionamiento.

La FEAADAH se erige como interlocutora con las instituciones del Estado para llegar adonde las asociaciones locales no alcanzan. Permite abordar una serie de necesidades que requieren una organización más compleja y que desbordan las posibilidades de una pequeña estructura horizontal. Su objetivo prioritario de actuación es trabajar en favor de la articulación de un marco institucional que favorezca la detección, diagnóstico y tratamiento del TDAH y que garantice el reconocimiento y respeto de los derechos de las personas afectadas. Tiene una actividad importante en Internet a través de su sitio web, su blog y cuentas en redes sociales en las que comparte noticias, informaciones o manifestaciones de su presidente frente a cuestiones que los afectan. Su adherencia al modelo biomédico vigente queda patente en los objetivos de la asociación, la información desplegada y la explicitación del comité de asesoramiento científico en el que se apoyan. Un análisis de la información contenida en su sitio web y en sus publicaciones nos permite destacar las principales características que definen a la FEAADAH en su función y en la relación con las políticas del conocimiento.

\section{La identidad colectiva}

El principal elemento aglutinador es el diagnóstico. Por ello, un apartado central del sitio web ofrece de manera detallada datos de carácter clínico sobre causas, detección, diagnóstico, trastornos asociados, tratamiento multimodal y consecuencias que podrían presentarse en su evolución. En su identidad pública y cohesionadora tratan de mantener un discurso estable y firme de una entidad nosológica que, de naufragar, los dejaría desposeídos de su medio de reconocimiento médico y articulación social. Por lo tanto, más que ofrecer información se ofrecen argumentos. Se privilegian las neuronarrativas $^{(37)}$ y las explicaciones genéticas que les permiten dejar un margen menor a la duda diagnóstica que si esta se expresa únicamente desde la apreciación clínica. La medicalización y el recurso a la ciencia en su discurso es el medio que les permite legitimar su experiencia ${ }^{(38)}$ y obtener recursos ${ }^{(36)}$, convertirse en interlocutores válidos de los expertos, no solo desde su conocimiento experiencial sino desde su experiencia de conocimiento experto, y fundamentar su discurso frente a la sociedad. Como afirma su presidente:

\begin{abstract}
Aprendimos a diferenciar el significado de términos como "metaanálisis", "ensayo clínico controlado ciego y doble ciego", "estudio longitudinal", "prevalencia, incidencia", "comorbilidad" y muchos otros, así como de variados instrumentos para elaborar y evaluar el conocimiento científico. Descubrimos que existe la denominada medicina basada en la evidencia, y que las revistas científicas de referencia de ámbito nacional e internacional cuentan con comités que establecen y controlan los estándares mínimos que deben cumplir los artículos que publican. [...] Hoy en las asociaciones somos conscientes de que, aun no siendo especialistas, tenemos el derecho, el deber y la capacidad para adquirir conocimiento sobre el TDAH y valorar la calidad de las fuentes en las que buscar información rigurosa. ${ }^{(39)}$
\end{abstract}

Esta identidad firme a partir del diagnóstico también tiene otros efectos. Por un lado, la relación con las asociaciones permite 
al sector biomédico refrendar su desempeño y hacer un mejor seguimiento del desarrollo del trastorno y los efectos del tratamiento. De hecho, la misma existencia de las asociaciones identificadas por la etiqueta diagnóstica se convierte en una evidencia basada en la experiencia. Por otro lado, permite los desmarques de madres y padres que no ven reconocidas sus experiencias ni expectativas en las fronteras del discurso biomédico dominante sobre el TDAH.

\section{Sensibilización}

A pesar de su reconocimiento médico e institucional, el debate sobre la existencia del TDAH continúa abierto en la sociedad. Un debate que, planteado en esos términos, considera que perjudica seriamente a las personas que sufren este trastorno y sus familias. En este sentido, las actividades de sensibilización juegan un papel determinante en la responsabilidad de las asociaciones para ser reconocidos por todos los profesionales que atienden a sus hijos y por la sociedad.

La sensibilización también pasa por la producción y difusión de conocimiento. Una de las prácticas discursivas utilizadas es el recurso estadístico de los datos y las cifras que permiten dimensionar el problema, por ejemplo, al mencionar que los pacientes con TDAH suponen cerca del $50 \%$ de la población clínica dentro de la psiquiatría infanto-juvenil. Otra es la recopilación de los mitos con los que socialmente se identifica el trastorno como invento, moda, mala educación, problemas de la vida moderna, entre otros, para proporcionar las respuestas verdaderas. Con la articulación de un discurso del riesgo también pretenden advertir sobre las consecuencias nefastas (drogas, alcoholismo, delincuencia) de los discursos negacionistas que obstaculizan la debida atención a la infancia con TDAH. Por último, con el propósito de liderar una propuesta de alcance internacional, en 2012 iniciaron una campaña para solicitar a las personas, especialmente las que desempeñan su actividad profesional en los ámbitos educativo, sanitario, social y laboral, que manifestaran su apoyo a la solicitud dirigida a la OMS para la declaración del Día Mundial de Sensibilización sobre el TDAH.

\section{Participación en la producción y movilización de conocimientos}

Gran parte de las actividades orbitan en torno a esta dimensión. En ellas se privilegian el conocimiento experto más que el experiencial, la objetividad derivada del rigor científico más que la subjetividad de las narrativas del malestar. Esto hace que, como han expresado en sus objetivos, promuevan actividades de formación e intercambio de proyectos, experiencias e investigaciones, así como la celebración de eventos que contribuyan a la divulgación e investigación sobre el TDAH. Asesoran tanto a madres y padres, como a profesionales, asociaciones e instituciones públicas y privadas sobre el TDAH. Ante una realidad compleja, trazan las hojas de ruta; $y$, ante el exceso informativo, recopilan información para garantizar la accesibilidad y la filtran en pos de la fiabilidad. También han mediado entre las instituciones sanitarias y las educativas, si bien de manera unidireccional, para tratar de expresar el sentido médico del trastorno.

Los conocimientos sobre el TDAH y su tratamiento no solo se erigen desde la afirmación sino desde la negación, el desmentido y la denuncia. Se difunden desde un "discurso del frente" con los que se responde a aquellas voces divergentes y no veraces que perjudican su tarea al banalizar el trastorno y sus causas. Hacer frente a los discursos contrarios pasa por una estrategia de deslegitimación en nombre de la ciencia. En una suerte de vigilancia epistemológica favorecida por las nuevas tecnologías, tanto los líderes de las asociaciones como sus asociados tienen una presencia muy activa en Internet y permanecen atentos a los desvíos del modelo narrativo biomédico o trivializaciones del trastorno que puedan producirse, para censurar, reconducir o demandar la asunción de responsabilidades a quienes corresponda. 
Un ejemplo de ello son las cartas abiertas del presidente de la Federación, los comentarios en blogs, periódicos o sitios web, a propósito de publicidades o informaciones que vayan en contra de sus intereses, o la recolección de firmas en el sitio web change.org. En una de las peticiones activas de esta última plataforma se solicita retirar de las carteleras una obra de teatro que atenta contra el TDAH y, en otra, la rectificación pública por parte de la dirección de un conocido programa de televisión en el que consideran que el trastorno se expuso de una forma totalmente parcial y errónea.

La FEAADAH recoge el discurso oficial que madres y padres hacen valer frente a las instituciones que no les cubren sus necesidades, los profesionales que no los respaldan y la sociedad que no los comprende ni respeta. Para ello, enarbolan y movilizan un discurso de certezas científicas en coherencia con los discursos expertos dominantes. Los grupos locales, que aglutina esta federación, presentan particularidades según los contextos locales en los que surgen, las personas que los nutren y sus trayectorias temporales. En una dimensión más micro, es posible observar cómo la solidez enunciativa que despliegan para que se reconozcan sus derechos es un artefacto discursivo que, en los grupos locales, dificulta la gestión de las distintas versiones del "nosotros".

\section{Asociación de alicante para el déficit de atención con o sin hiperactividad (AADAH): prácticas situadas y discursos en los márgenes}

A diferencia del carácter formal y aséptico de la federación, los grupos locales conservan algunos trazos de los grupos de ayuda mutua en los que la equivalencia y la confianza se construyen sobre la identificación basada en la historia compartida de una misma trayectoria, azarosa e incierta, y de un mismo problema. A partir de aquí, el grupo define su propia identidad, resignifica el problema compartido y hace posible la reconstrucción de la identidad deteriorada de sus miembros, e incluso su reformulación sobre otros parámetros ${ }^{(40)}$. Así, en 2012, un grupo de madres y padres que coincidían con sus hijos en la Unidad de Salud Mental Infanto-Juvenil, vieron la necesidad de poner en marcha la asociación para la mejora de la situación de los niños y las familias que padecen TDAH y la defensa de sus derechos.

Entre sus objetivos se repiten aquellos Ilamados a paliar el desconocimiento social sobre el trastorno, la difusión de su comprensión en los ámbitos educativo, sanitario, social y familiar y la defensa de sus derechos en las administraciones locales. De ahí que sus principales actividades sean los talleres educativos, charlas o ponencias, el asesoramiento a las familias en la detección precoz y el tratamiento multidisciplinario, y la representación colectiva ante los distintos organismos. La corta andadura de este grupo de apoyo y su discreto tamaño hace que no tengan profesionales en plantilla, aunque sí expertos colaboradores de referencia que imparten las charlas y los asesoran.

Su carácter de proximidad y cercanía cumple una labor de apoyo emocional indispensable para aquellos padres que, buscando sus iguales en experiencia, Ilegan desorientados con la etiqueta médica o con la sospecha de ella demandando información y orientación. No obstante, quienes mantienen el vínculo y establecen una relación de ayuda mutua y se involucran con la asociación son una minoría frente a aquellos asociados que cumplen más un perfil de consumo de servicios e información sobre recursos educativos y sanitarios locales.

Son especialmente sensibles al trato que reciben de los médicos y al que reciben sus hijos en las escuelas cuando cuestionan el TDAH. De hecho, uno de los elementos que homologa sus experiencias es precisamente el (des)conocimiento social sobre el trastorno. "Compartir un mismo problema" precisa que se comparta "una historia de un mismo problema", una historia que no es reducible a la anamnesis, sino que abarca las vivencias, experiencias y circunstancias asociadas al problema, incluida la experiencia de relación con los profesionales y los servicios ${ }^{(41)}$. Como afirma la presidenta de la asociación: 
Hay madres que me han venido a decir "es que yo no paro de decirle a mi pediatra que yo veo esto y veo lo otro, pero me dicen que soy una madre muy exagerada, una histérica, que cómo va a ser hiperactivo, que eso no, que eso nada y no me manda donde me tiene que mandar". Entonces, no es solamente en educación, también en la sanidad. (Madre de dos hijos con TDAH, presidenta de una asociación)

También sienten la presión de un entorno que critica sus estilos parentales:

...lo que normalmente los padres tienen que oír es que ese niño no está bien educado, que sus padres no le ponen límites, que ese niño es malo o vago, o que ese niño está en las nubes. Entonces, claro, se va a culpabilizar al niño y a la familia de un trastorno en el que la familia tiene mucho que hacer, por supuesto, y el trabajo con el niño es fundamental, pero es que es algo neurobiológico y que los padres no pueden controlar desde que el niño es pequeño. (Madre de dos hijos con TDAH, presidenta de una asociación)

En este sentido, recibir el diagnóstico es tranquilizador, dado que les permite matizar la culpa por la crianza, orientar la búsqueda de recursos e identificar a los profesionales que los pueden ayudar. El énfasis en que el diagnóstico explica lo incontrolable tiene un efecto expiatorio que los exime de la responsabilidad, los absuelve de culpa y preserva su identidad como buenos padres ${ }^{(3,21)}$. No obstante, lamentan que no haya una prueba diagnóstica que fuera más clara para legitimar su relato de manera irrefutable: "Ojalá tuviéramos, el análisis de sangre para medir la 'hiperactivina' y la 'impulsivina' y todo esto, ¿no?"

La alineación con el discurso experto dominante lleva a una homogeneización del relato que no está exenta de conflictos intragrupales, dado que obliga a la convergencia de la explicación biomédica vigente como autocensura estratégica de los grupos, en detrimento del conocimiento experiencial que se hace valer, pero al margen de las actividades grupales. En este sentido, la presidenta afirmaba la dificultad de organizar actividades que respondieran a los intereses de todos, aunque ella misma excediera los límites de los tratamientos que la biomedicina reconoce:

Por una parte tienes las personas que "yo voy a mi psiquiatra, voy a mi pediatra, voy a mi psicólogo, y con esto el niño pues va mejorando". A esas personas no les digas: "ven a una charla de flores de Bach y de terapia cromatográfica o de homeopatía". Porque van a decir: "vaya porquería, eso es pseudociencia, eso no es nada" [...] Pero, por ejemplo, yo he llevado a mis hijos a un homeópata, que he empezado ahora, y voy a dar una oportunidad a eso [...] En mi caso, mi marido y yo preferiríamos no medicar. [...] Es difícil también lidiar con toda la gama de personas que hay dentro de una asociación para que todas encuentren una respuesta que les valga dentro de la asociación. Es decir, si yo ahora mismo empiezo a hacer charlas de tapping, de flores de Bach, la mitad de los socios se me va a ir, o la gran mayoría. En cambio, la otra parte estarán encantados. Entonces, hay que dar cabida a todo el mundo y eso es complicado. (Madre de dos hijos con TDAH, presidenta de una asociación)

Los paradigmas se mantienen o cambian según efectos de la medicación ${ }^{(38)}$, por ello hay un proceso de contraste entre el discurso oficial y la experiencia, en el que se toman decisiones no informadas sino experimentadas que generan una hibridación del conocimiento experto y alterexperto. Pero lo que queda patente es que sus complejos asistenciales desbordan los límites biomédicos previstos, en tanto que el ámbito en el cual los actores sociales implicados ${ }^{(42)}$ se piensan, se representan simbólicamente y despliegan sus decisiones va más allá de las fronteras 
epistémicas del discurso acreditado. Sin embargo, este ha de permanecer blindado para preservar la identidad colectiva que les permita defender sus derechos. Precisamente cuando estas fronteras no permiten otras narrativas que expliquen la hiperactividad $u$ otras opciones terapéuticas es cuando aparecen los exilios grupales y la activación de otros marcos de sentido, como veremos en el caso que sigue.

\section{Yo amo a alguien con... ¿TDAH?: singularidades en red}

Si has Ilegado hasta aquí seguro que alguien cercano a ti ha sido etiquetado con las siglas TDAH. Nuestro hijo también, por esa razón empezamos con este blog para recopilar y compartir toda la información con la que nos íbamos encontrando. Después de este tiempo nos ha servido de mucho más. Hemos dado un nuevo rumbo al tratamiento de nuestro hijo, algo que hubiese sido imposible sin las personas que hemos conocido durante el camino y las alternativas que hemos descubierto que existían, aunque nadie antes nos hablara de ellas. Nuestro objetivo ahora es que nuestras experiencias os sirvan para sacar vuestras propias conclusiones y podáis elegir la manera de ayudar a vuestr@hij@ desde una óptica más abierta. ${ }^{(43)}$

Con esta invitación, el autor del seguido blog Yo amo a alguien con... ¿TDAH? se presenta a los lectores que buscan información sobre el TDAH. Comenzó el blog en 2009, a modo de "biblioteca", como una manera de organizar lo que iba leyendo en su proceso de autoformación y búsqueda de respuestas alternativas a las recibidas en el circuito de médicos, psicólogos y asociaciones que había conocido, para quienes no cabía duda de que su hijo tenía TDAH y que el único tratamiento disponible era la terapia cognitivoconductual y la medicación. Precisamente, los efectos de la medicación en la expresión del rostro irreconocible de su hijo fueron los que determinaron que abandonara ese camino y buscara otras respuestas y otros recursos para entender qué le sucedía.

En su caso, las experiencias asociativas no fueron satisfactorias, justamente por los escasos márgenes para moverse en otros referentes de conocimiento. Empezó acudiendo a la asociación más cercana pero le cuestionaban sus comentarios, aduciendo la disciplina científica desde la cual hablaba. Su posicionamiento no es contra la ciencia sino contra las versiones únicas y estandarizadas de que exista solo una posibilidad explicativa para todos, que no deja margen a la experiencia del ensayo-error:

Yo soy geólogo de carrera, soy científico todo lo que puedo. Pero llega un momento que dices: "es que si sigo intentando ceñirme tanto a la ciencia, me cierro muchas puertas". Y no pasa nada por abrirlas y luego ver que no $y$ cerrarlas. (Padre de un hijo con TDAH, autor de un blog)

En el periplo de su iniciativa ha pasado del privilegio a la biosocialidad como modelo explicativo, al privilegio epistémico de la biografía, que le llevó a poner los interrogantes al TDAH que aparecían en el título de su blog:
A mí me han hecho perder tiempo los médicos tremendamente. Porque el niño tenía un problema en el colegio y los médicos saben que lo que le pasa al mío podrían haber llegado a esas cosas si se hubieran esforzado un pelín, ¿sabes? Y no hubieran menospreciado la infor- mación que yo les he dado. Por ejem- plo, el tema de la adopción y tal. Que lo obvian, así como si nada, y eso tiene muchas repercusiones y se saben. (Padre de un hijo con TDAH, autor de un blog)

Asimismo, a modo de estructura rizomática, se ha ido creando una red de expertos disidentes y asociaciones, unidos por el cuestionamiento del estatus actual del TDAH desde una variedad de discursos, lugares 
de enunciación y estrategias de atención. Algunos de estos grupos son: "Padres con alternativas", "Asociación Laztana", "Cuando no todos son TDAH" o "Ventaja por déficit de atención con hiperactividad".

\begin{abstract}
Dice un amigo mío psiquiatra que me ha seguido desde el principio que para él es muy interesante ver cómo he evolucionado. Primero viendo todo lo que había, abriendo puertas aquí y allá, en todo -lo bueno y lo malo-, con lo que estaba de acuerdo y lo que no, y luego ponerte en contra completamente de todos los científicos: de los psiquiatras, de los médicos. Y luego tener tu propia opinión que ya, por suerte, no es solo la mía. Ahí me ha costado años encontrar el "corrillo". (Padre de un hijo con TDAH, autor de un blog)
\end{abstract}

La construcción de una narrativa compartida y consensuada permite que otros grupos que confrontan el discurso institucionalizado produzcan una forma de Conocimiento cuya legitimidad, basada en la especificidad de la experiencia, puede ayudar a confrontar los discursos dominantes ${ }^{(21)}$. Esto muestra la capacidad de Internet como entorno para crear comunidades de proximidad y apoyo desencarnadas, en las que la formación y difusión del conocimiento permiten la transformación personal y COlectiva; y ofrece posibilidades de acción colectiva cuando los caminos y recursos más recurrentes no responden a las expectativas, cuando no hay a quién asociarse ni se sabe qué hacer:

Esa es la peor sensación que he tenido en este tema. Que te quedas absolutamente solo. Nadie te dice nada. Si tú te sales de la vía convencional, nadie te dice nada. (Padre de un hijo con TDAH, autor de un blog)

Cuando no se reconoce la etiqueta vigente, no hay siglas bajo las que agruparse. El autor de este blog es el ejemplo de una singularidad interconectada y movilizadora de un alterconocimiento gestado diacrónicamente, alternativo a las asociaciones mayoritarias.

\section{CONCLUSIONES}

El reconocimiento diagnóstico es fundamental en un contexto de disenso pues permite una articulación burocrática, social y administrativa en los sistemas de salud de los estados de bienestar modernos. El énfasis en el criterio diagnóstico y el proceder biomédico es más señalado en aquellos países, como España, en los que la opinión pública y experta no ha legitimado los discursos biomédicos sobre la hiperactividad. La concepción diagnóstica y las opciones terapéuticas son los principales nodos de encuentro o puntos de fuga que establecen la doble frontera entre expertos y legos, lo que hace del conocimiento el principal dominio en el que se piensan, identifican y articulan como colectivos o singularidades, en los que la legitimación de unas voces deslegitima otras.

Las posiciones, aunque con matices, se pueden reducir, por un lado, a la adhesión de un sector mayoritario de madres y padres, agrupados en la FEAADAH, al diagnóstico y al conocimiento experto biomédico que lo valida, utilizando la medicalización como medio de articulación social para conseguir mejoras en el bienestar de los hijos. Mientras que, por otro lado, estarían las experiencias del exilio, como el autor del blog, que si en un inicio se identifica con la etiqueta, el conocimiento de otros discursos que la rechazan va a implicar la articulación de otras redes de apoyo ex novo en entornos más amplios y desencarnados como Internet. De estos posicionamientos se derivan las distintas estrategias de participación en salud de cada grupo.

En un caso de controversias científicas como la que nos ocupa, la movilización de recursos y rearticulación de saberes en las asociaciones implican un estado de vigilancia epistémica y una participación muy activa para que el discurso no se deforme, ni pierda legitimidad. Los grupos de apoyo pasan a 
ser grupos de presión en su forma federada, participando en la toma de decisiones sanitarias y educativas, y en la reivindicación de derechos y ciudadanía biosocial.

En el caso de las estrategias de producción y colectivización de conocimientos, facilitadas por Internet, observamos cómo se privilegian las narrativas de la experiencia epistémica y su amplificación en las redes sociales, pasando del qué tengo al cómo lo gestiono. Esta estrategia permite el acceso a la información, la construcción colectiva de conocimiento y una gestión más flexible del vínculo personal y geográfico; aquí, el discurso experiencial atrae la adherencia de otros grupos y expertos, mientras que en las asociaciones, las adherencias biomédicas secuestran el discurso de la experiencia.
Las características del TDAH como entidad médica con una prevalencia significativa, pero falta de consenso social y profesional, ofrece un panorama más plural que la relación bidireccional entre legos y expertos que, por un lado, da por hecho la homogeneidad del discurso lego y, por otro, pone su atención en la institucionalización de la experiencia, dejando puntos ciegos a otras movilizaciones. Este trabajo ha tratado de contextualizar las iniciativas parentales en torno al TDAH en España, no solo atendiendo a la estructura asociativa más visible y al discurso preponderante, sino contemplando otras estrategias de activismo en salud al margen de las asociaciones y poniéndolas en relación con los distintos debates abiertos.

\section{AGRADECIMIENTOS}

Al Ministerio de Educación y Cultura de España por la financiación del proyecto de investigación del que forma parte este trabajo "Visiones y versiones de las tecnologías biomédicas. Análisis de la producción y circulación de saberes expertos/legos en prácticas biomédicas" (FFI201238912-C02-02). Asimismo, deseo expresar mi gratitud hacia las personas que han colaborado en esta investigación prestándome su tiempo, experiencias y saberes.

\section{REFERENCIAS BIBLIOGRÁFICAS}

1. García Peñas J, Domínguez Carral J. ¿Existe un sobrediagnóstico del trastorno de déficit de atención e hiperactividad (TDAH)? Evidencias en Pediatría. 2012;8:51.

2. Lange KW, Reichl S, Lange KM, Tucha L, Tucha $\mathrm{O}$. The history of attention deficit hyperactivity disorder. ADHD Attention Deficit and Hyperactivity Disorders 2010;2(4):241-255.

3. Brinkmann S. Diagnostic cultures: A cultural approach to the pathologization of modern life. London: Routledge; 2016.

4. Edwards C, Howlett E, Akrich M, Rabeharisoa V. Attention deficit hyperactivity disorder in France and Ireland: parents' groups' scientific and political framing of an unsettled condition. BioSocieties. 2014;9(2):153-172.
5. Singh I. Beyond polemics: science and ethics of ADHD. Nature Reviews Neuroscience. 2008;9(12): 957-964.

6. Martínez Hernández A. La mercantilización de los estados de ánimo: El consumo de antidepresivos y las nuevas biopolíticas de las aflicciones. Política y Sociedad. 2006;43(3):43-56.

7. American Psychiatric Association. Diagnostic and statistical manual of mental disorders. 5th ed. Arlington: APA; 2013.

8. Cohen S. The mental hygiene movement, the development of personality, and the school: The medicalization of American education. History of Education Quarterly. 1983;23(2):123-149.

9. Singh I. A framework for understanding trends in ADHD diagnoses and stimulant drug treatment: schools and schooling as a case study. BioSocieties. 2006;1(04):439-452.

10. Kristjánsson K. Medicalised pupils: the case of ADD/ADHD. Oxford Review of Education. 2009;35(1):111-127.

11. Polanczyk G, de Lima MS, Horta BL, Biederman J, Rohde LA. The worldwide prevalence of ADHD: a systematic review and metaregression analysis. The American Journal of Psychiatry. 2007; 164(6):942-948.

12. Wittchen $H$, Jacobi $F$, Rehm J, Gustavsson A, Svensson M, Jönsson B, et al. The size and burden of mental disorders and other disorders of the brain 
in Europe 2010. European Neuropsychopharmacology. 2011;21(9):655-679.

13. Catalá-López F, Peiró S, Ridao M, SanfélixGimeno G, Gènova-Maleras R, Catalá MA. Prevalence of attention deficit hyperactivity disorder among children and adolescents in Spain: a systematic review and meta-analysis of epidemiological studies. BMC Psychiatry. 2012;12:168.

14. Canals Sala J. El regreso de la reciprocidad: Grupos de ayuda mutua y asociaciones de personas afectadas en la crisis del Estado del Bienestar. Tarragona: Universitat Rovira i Virgili; 2003.

15. Rabeharisoa V, Callon M. La participación de las asociaciones de pacientes en la investigación. Revista Internacional de Ciencias Sociales. [Internet] 2002;171 [citado 1 oct 2016]. Disponible en: http://www.oei.es/salactsi/volona.pdf

16. Wehling $\mathrm{P}$, Viehöver $\mathrm{W}$, Koenen $\mathrm{S}$, (eds.). The public shaping of medical research: patient associations, health movements and biomedicine. London: Routledge; 2014.

17. García Dauder S. La regulación tecnológica del dualismo sexual y el diseño de cuerpos normativos. En: Pérez Sedeño E, Ortega E. Cartografías del cuerpo: biopolíticas de la ciencia y la tecnología. Valencia: PUV; 2014. p. 469-520.

18. Ortega Arjonilla E, Romero Bachiller C, Ibáñez Martín R. Discurso activista y estatus médico de lo trans: hacia una reconfiguración de cuidados y diagnósticos. En: Pérez Sedeño E, Ortega E. Cartografías del cuerpo: biopolíticas de la ciencia y la tecnología. Valencia: PUV; 2014. p. 521-572.

19. Gregori N. 'Llegar a ser' mujer/hombre desde un diagnóstico de intersexualidad o ADS. En: Pérez Sedeño E, Ortega E. Cartografías del cuerpo: biopolíticas de la ciencia y la tecnología. Valencia: PUV; 2014. p. 423-468.

20. Dauder SG, Flor NG, García IH. Usos de lo psicosocial en la investigación y tratamiento de las intersexualidades/DSD. Universitas Psychologica. 2015;14(5):1649-1666.

21. Frigerio A, Montali L. An ethnographic-discursive approach to parental self-help groups: The case of ADHD. Qualitative Health Research. 2016;26(7):935-950.

22. Gray Brunton C, McVittie C, Ellison M, Willock J. Negotiating parental accountability in the face of uncertainty for attention-deficit hyperactivity disorder. Qualitative Health Research. 2014;24(2):242-253.
23. Singh I. Boys will be boys: Fathers' perspectives on ADHD symptoms, diagnosis, and drug treatment. Harvard Review of Psychiatry. 2003;11(6):308-316.

24. Conrad P, Potter D. From hyperactive children to ADHD adults: Observations on the expansion of medical categories. Social Problems. 2000;47(4):559-582.

25. Phillips CB. Medicine goes to school: teachers as sickness brokers for ADHD. PLoS Medicine. 2006;3(4):e182.

26. Mabres M, (ed.). Hiperactividades y déficit de atención: comprendiendo el TDAH. Barcelona: Octaedro; 2013.

27. Malacrida C. Medicalization, ambivalence and social control: mothers' descriptions of educators and ADD/ADHD. Health (London). 2004;8(1):61-80.

28. Graham LJ. From ABCs to ADHD: the role of schooling in the construction of behaviour disorder and production of disorderly objects. International Journal of Inclusive Education. 2008;12(1):7-33.

29. Rodríguez CJG. Creciendo juntos: de la infancia a la adolescencia con cariño y respeto. Madrid: Temas de Hoy; 2013.

30. García de Vinuesa F, González Pardo H, Pérez Álvarez M. Volviendo a la normalidad: La invención del TDAH y del trastorno bipolar infantil. Madrid: Alianza editorial; 2014.

31. Jara M. Traficantes de salud: cómo nos venden medicamentos peligrosos y juegan con la enfermedad. Barcelona: Icaria Editorial; 2007.

32. Grupo de trabajo de la Guía Práctica Clínica sobre el Trastorno por Déficit de Atención con Hiperactividad (TDAH) en Niños y Adolescentes, Fundació Sant Joan de Déu, (coords.). Guía de práctica clínica sobre el trastorno por déficit de atención con hiperactividad (TDAH) en niños y adolescentes. Cataluña: Ministerio de Sanidad, Política Social e Igualdad, AIAQS; 2010.

33. Rafalovich A. Psychodynamic and neurological perspectives on ADHD: Exploring strategies for defining a phenomenon. Journal for the Theory of Social Behaviour. 2001;31(4):397-418.

34. Southall A. The other side of ADHD: Attention deficit hyperactivity disorder exposed and explained. Abingdon: Radcliffe Publishing; 2007.

35. Haas PM. Introduction: epistemic communities and international policy coordination. International Organization. 1992;46(1):1-35. 
36. Edwards C, Howlett E, Akrich M, Rabeharisoa $\mathrm{V}$. Attention deficit hyperactivity disorder in France and Ireland: parents' groups' scientific and political framing of an unsettled condition. BioSocieties. 2014;9(2):153-172.

37. Hernáez ÁM. La mecantilización de los estados de ánimo: el consumo de antidepresivos y las nuevas biopolíticas de las aflicciones. Política y Sociedad. 2006;43(3):43-56.

38. Bröer C, Heerings M. Neurobiology in public and private discourse: the case of adults with ADHD. Sociology of Health \& Illness. 2013;35(1):49-65.

39. Conesa FM. Un debate artificial. Cuadernos de Pedagogía. 2016;(463):82-87.
40. Sala JC. Grupos de ayuda mutua y asociaciones de personas afectadas: reciprocidades, identidades y dependencias. Cuadernos de Psiquiatría Comunitaria. 2003;3(1):71-81.

41. Frigerio A, Montali L, Fine M. Attention deficit/ hyperactivity disorder blame game: a study on the positioning of professionals, teachers and parents. Health (London). 2013;17(6):584-604.

42. Comelles JM. De la ayuda mutua y de la asistencia como categorías antropológicas: Una revisión conceptual. Trabajo Social y Salud. 2000;35:151-172.

43. Badia J. Yo amo a alguien con... iTDAH? [Internet]. c2017 [citado 10 oct 2016]. Disponible en: http://tinyurl.com/ycnkqfkb.

FORMA DE CITAR

Hurtado García I. Asociaciones y disociaciones: agentes, discursos y controversias en torno a la hiperactividad infantil. Salud Colectiva. 2017;13(2):321-335. doi: 10.18294/sc.2017.1212.

Recibido: 1 de noviembre de 2016 | Aprobado: 2 de marzo de 2017

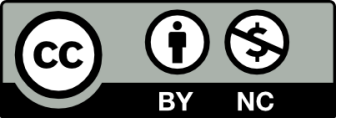

Este obra está bajo una licencia de Creative Commons Reconocimiento-NoComercial 4.0 Internacional. Reconocimiento - Permite copiar, distribuir y comunicar públicamente la obra. A cambio, se debe reconocer y citar al autor original. No Comercial - Esta obra no puede ser utilizada con finalidades comerciales, a menos que se obtenga el permiso. 